\title{
Gustavo Olague: Evolutionary computer vision, the first footprints
}

\author{
Springer, 2016, 400 pp, ISBN: 978-3-662-43692-9
}

\author{
Evelyne Lutton ${ }^{1}$
}

(C) Springer Science+Business Media, LLC 2017

For about 20 years, evolutionary computation (as it provides a useful set of tools to deal with hard real-world problems) has been exploited in image processing and computer vision. This has lead to the establishment of an emergent research topic named evolutionary computer vision (ECV). Olague's book aims to lay the foundations of this interdisciplinary research topic, concentrating particularly on goal-driven vision.

To my mind, Dr. Gustavo Olague is certainly among the first and most active researchers of the evolutionary computer vision domain. He is Professor of Computer Science at CICESE in Tijuana, Mexico, where he founded the EvoVision group research group, which is dedicated to ECV. His background is in computer vision. He obtained his Ph.D. at the French National Polytechnic Institute of Grenoble associated with an INRIA $^{1}$ research team, renowned for his high quality research in robotic vision. Unlike most ECV researchers, who are experts in stochastic optimisation and who address image processing and robot vision problems as challenging applications of their favourite evolutionary optimisation algorithms, Gustavo Olague is among the very few people able to have a foot in both optimisation and in computer vision.

The development of artificial vision systems has been based on various analogies with biology and nature-inspired paradigms (stereovision or pattern recognition, for instance). Natural evolution is another source of inspiration and the main topic this book. But with an additional pinch of salt: natural vision systems are the result of millennia of evolution. So we may expect they are optimal in some sense, still to be unravelled. In this book, through questioning in a theoretical, philosophical, and

\footnotetext{
Evelyne Lutton

evelyne.lutton@inra.fr

1 INRA, Grignon, France
}

1 INRIA is the french institute for research in computer science. 
practical way the purpose of artificial vision systems, Gustavo Olague is designing a framework to guide future research in ECV.

Darwinian principles have been used to build robust optimisers (e.g. evolutionary strategies, genetic algorithms and genetic programming), which have been successfully applied in many domains. For ECV, the major issue has been to formulate artificial vision problems in terms that are suitable for optimisation, hence the notion of goal-driven vision. This way of formulating problems, not common in computer vision, is a brake on the development of ECV. The book puts goal-driven vision in perspective by Gustavo Olague questioning the teleology (from the Greek telos, "purpose") of natural evolution. For the moment, artificial vision systems rely on very simplified, computable processes, but it seems obvious now that a careful design of complex goals for vision systems may yield new achievements.

This book is very pleasant to read, clear and well explained. Thanks to introductory chapters, it is accessible to students and beginners in both robot vision and evolutionary computation. At the same time it is not boring for domain experts: Dr. Olague dots the text with original viewpoints, historical perspectives, and philosophical arguments. He also provides rigorous mathematical and pedagogical explanations of various important notions.

The glowing foreword by Marco Tomasini tells everything about the book. The book itself reviews the history and challenging issues of both computer vision and evolutionary computation in the first chapters. The rest of the book provides detailed and convincing examples derived from Gustavo Olague's own experience at his research lab, EvoVision. The text is organised into low, mid and high level tasks, reflecting the classical taxonomy for vision tasks, and representing at the same time a progression in terms of the complexity of the fitness function and goal formulation tasks.

I especially liked the pages dedicated to the design of evolutionary computation (pp. 114-116). This is a major issue for the design of efficient applications. The goal is not only embedded into the function to be optimised (fitness function and possible constraints) but also into the whole problem formulation: how the search space is represented (solution encoding), and explored (evolutionary operators).

Special mention should be made on the use of multi-objective and cooperationcoevolution techniques for dealing with complex vision tasks. As they allow additional freedom for algorithmic design, these "non-standard" exploitation of natural evolution principles are very promising in many domains and in particular in artificial vision. They also map a route to automated and interactive problem design. Although out of the scope of the book, they are sketched in the concluding chapter. If human-competitive results now exist in ECV human-collaborative perspectives may open new research challenges.

In conclusion, this excellent book is suitable for a large audience: beginners will find all the important features to start with, while specialists will find food for thought. 